\title{
Quantum field theory with no zero-point energy
}

\section{John R. Klauder}

Department of Physics, University of Florida, Gainesville, FL 32611-8440, U.S.A.

Department of Mathematics, University of Florida, Gainesville, FL 32611-8440, U.S.A.

E-mail: john.klauder@gmail.com

ABSTRACT: Traditional quantum field theory can lead to enormous zero-point energy, which markedly disagrees with experiment. Unfortunately, this situation is built into conventional canonical quantization procedures. For identical classical theories, an alternative quantization procedure, called affine field quantization, leads to the desirable feature of having a vanishing zero-point energy. This procedure has been applied to renormalizable and nonrenormalizable covariant scalar fields, fermion fields, as well as general relativity. Simpler models are offered as an introduction to affine field quantization.

Keywords: Nonperturbative Effects, Renormalization Regularization and Renormalons, Models of Quantum Gravity

ARXiv EPrint: 1803.05823 


\section{Contents}

1 Introduction 1

2 Nonrenormalizable models exposed 2

3 Ultralocal scalar model: a nonrenormalizable example $\quad 3$

4 Additional studies using affine variables 5

\section{Introduction}

The source of zero-point energy for a free scalar field is readily canceled by normal ordering of the Hamiltonian operator, which leaves every term with an annihilation operator. Unfortunately, for a non-free scalar field, say with a quartic potential term, normal ordering cannot eliminate the zero-point energy since there is always a term composed only of creation operators.

This situation is inevitable with canonical quantization (CQ) when a classical field $\phi(x) \rightarrow \hat{\phi}(x)=\left[A(x)^{\dagger}+A(x)\right] / \sqrt{2}$, where $x \in \mathbb{R}^{s}, s \in\{1,2,3, \ldots\}$, and its momentum field $\pi(x) \rightarrow \hat{\pi}(x)=i \hbar\left[A(x)^{\dagger}-A(x)\right] / \sqrt{2},\left[A(x), A(y)^{\dagger}\right]=\delta(x-y) \mathbb{1}$, and $[\hat{\phi}(x), \hat{\pi}(y)]=$ $i \hbar \delta(x-y) \mathbb{1}$. As usual, the ground state of the Hamiltonian, designated by $|0\rangle$, ideally is the unique, normalized, 'no particle' state for which $A(x)|0\rangle \equiv 0$ for all $x$, and Hilbert space is given by suitable combinations of terms such as $A\left(x_{1}\right)^{\dagger} A\left(x_{2}\right)^{\dagger} \cdots A\left(x_{N}\right)^{\dagger}|0\rangle$ for all $N<\infty$ when smeared with suitable elements $f\left(x_{1}, \ldots, x_{N}\right) \in L^{2}\left(\mathbb{R}^{s N}\right)$.

However, there is also a different approach. In place of the momentum, we substitute the dilation field $\kappa(x) \equiv \pi(x) \phi(x)$ - a field that is featured in the enhanced quantization (EQ) program [1] — which leads to the Poisson bracket $\{\phi(x), \kappa(y)\}=\delta(x-y) \phi(x)$, a formal Lie algebra for the affine group. As the analog of a current commutation relation [2], an affine quantization of these variables leads to $\phi(x) \rightarrow \hat{\phi}(x) \equiv \int B(x, \lambda)^{\dagger} \lambda B(x, \lambda) d \lambda$ and $\kappa(x) \rightarrow \hat{\kappa}(x) \equiv \int B(x, \lambda)^{\dagger} \tau(\lambda) B(x, \lambda) d \lambda$, where $\tau(\lambda) \equiv-\frac{1}{2} i \hbar[\lambda(\partial / \partial \lambda)+(\partial / \partial \lambda) \lambda]$. Here $B(x, \lambda) \equiv A(x, \lambda)+c(\lambda) \mathbb{1}$, where $\lambda \in \mathbb{R}, A(x, \lambda)|0\rangle=0$ for all $(x, \lambda)$, and $c(\lambda)$ is the real 'model function'; for the example offered in section $3 c(\lambda)=|\lambda|^{-1 / 2} W(\lambda)$, where $0<W(\lambda)=W(-\lambda)<\infty$ with $0<\epsilon<W(0)$. Local operator products are given by an operator product expansion [2], which leads to renormalized (R) products such as

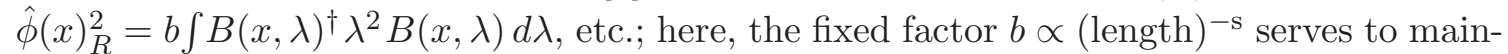
tain proper dimensions. Such local products become terms in the quantum Hamiltonian, which is given by

$$
\begin{aligned}
\mathfrak{H} & \equiv \int B(x, \lambda)^{\dagger} \mathfrak{h}(\partial / \partial \lambda, \lambda) B(x, \lambda) d \lambda d^{s} x \\
& =\int A(x, \lambda)^{\dagger} \mathfrak{h}(\partial / \partial \lambda, \lambda) A(x, \lambda) d \lambda d^{s} x,
\end{aligned}
$$


which requires that $\mathfrak{h}(\partial / \partial \lambda, \lambda) c(\lambda)=0$. For the example of section 3, which has a classical Hamiltonian density of $\frac{1}{2} \pi(x)^{2}+V(\phi(x))$, for some $V$ with no spatial derivatives, then, with $\hbar=1$,

$$
\begin{aligned}
\mathfrak{h}(\partial / \partial \lambda, \lambda) & =-c(\lambda)^{-1} \partial / \partial \lambda c(\lambda)^{2} \partial / \partial \lambda c(\lambda)^{-1} \\
& =-\partial^{2} / \partial \lambda^{2}+c(\lambda)^{-1} \partial^{2} c(\lambda) / \partial \lambda^{2} .
\end{aligned}
$$

We require that $\int\left[\lambda^{2} /\left(1+\lambda^{2}\right)\right] c(\lambda)^{2} d \lambda<\infty$, and, to obtain a unique ground state, we insist that $\int c(\lambda)^{2} d \lambda=\infty$, which ensures that $\mathfrak{h}>0$, as normalized expectations of the top line of (1.2) would confirm.

Hence: (i) $\mathfrak{H} \geq 0$, (ii) $\mathfrak{H}$ has a unique ground state $|0\rangle$ (because $c(\lambda) \notin L^{2}(\mathbb{R})$ implies that all eigenvalues of $\mathfrak{h}$ with normalizable eigenfunctions are positive), and (iii) $\mathfrak{H}$ has no zero-point energy (because all terms in $\mathfrak{H}$ lead with an annihilation operator). The Hilbert space has combinations of $A\left(x_{1}, \lambda_{1}\right)^{\dagger} A\left(x_{2}, \lambda_{2}\right)^{\dagger} \cdots A\left(x_{N}, \lambda_{N}\right)^{\dagger}|0\rangle$ for all $N<\infty$ when smeared with suitable elements $f\left(x_{1}, \lambda_{1}, \ldots, x_{N}, \lambda_{N}\right) \in L^{2}\left(\mathbb{R}^{(s+1) N}\right)$.

\section{Nonrenormalizable models exposed}

A simple 'toy' example can help understand nonrenormalizable models. Consider the onevariable problem with a classical action functional given by $I_{g}=\int_{0}^{T}\left[\frac{1}{2} \dot{q}(t)^{2}-\frac{1}{2} q(t)^{2}-\right.$ $\left.g q(t)^{-4}\right] d t$, where the coupling constant $g \geq 0$. The free model, with $g=0$, has a domain of functions $D_{g=0}=\left\{q: \int_{0}^{T}\left[\dot{q}^{2}+q^{2}\right] d t<\infty\right\}$, while the interacting theory, with any $g>0$, has a domain of functions $D_{g>0}=\left\{q: \int_{0}^{T}\left[\dot{q}^{2}+q^{2}+q^{-4}\right] d t<\infty\right\}$. Clearly, $D_{g>0} \subset D_{g=0}$, and, in particular, while solutions to the equations of motion within $D_{g=0}$ cross back and forth over $q=0$, solutions to the equations of motion within $D_{g>0}$ never cross $q=0$ and are always positive or always negative. In fact, solutions to the equations of motion when $g>0$ find that the limiting behavior of such solutions as $g \rightarrow 0$ is continuously connected to a solution that belongs to $D_{g>0}$ and does not belong to $D_{g=0}$. Specifically, a free model solution is given by $q(t)=B \cos (t+\beta)$, for any $B$ and $\beta$, while a $g \rightarrow 0$ solution is given by $q(t)= \pm|B \cos (t+\beta)| \neq 0$. In brief, as $g \rightarrow 0$, an interacting model is continuously connected with the free action functional but with the constraint that its associated domain is $D_{g>0}$. Thus, the 'interacting models' are not continuously connected to the 'free model'! We say that the $g \rightarrow 0$ solutions belong to a pseudofree model rather than the free model.

Quantum mechanically, the propagator for the free model is given by

$$
K_{f}\left(q^{\prime \prime}, T ; q^{\prime}, 0\right)=\Sigma_{n=0,1,2,3, \ldots} h_{n}\left(q^{\prime \prime}\right) h_{n}\left(q^{\prime}\right) e^{-i(n+1 / 2) T / \hbar},
$$

where $\left\{h_{n}(q)\right\}_{n=0}^{\infty}$ are Hermite functions. On the other hand, the propagator for the pseudofree model [3] is given by

$$
\begin{aligned}
K_{p f}\left(q^{\prime \prime}, T ; q^{\prime}, 0\right) & =\lim _{g \rightarrow 0} \mathcal{N}_{g} \int_{q(0)=q^{\prime}}^{q(T)=q^{\prime \prime}} e^{(i / \hbar) \int_{0}^{T}\left[\left(\dot{q}^{2}-q^{2}\right) / 2-g q^{-4}\right] d t} \mathcal{D} q \\
& =2 \theta\left(q^{\prime \prime} q^{\prime}\right) \Sigma_{n=1,3,5,7 \ldots} h_{n}\left(q^{\prime \prime}\right) h_{n}\left(q^{\prime}\right) e^{-i(n+1 / 2) T / \hbar}
\end{aligned}
$$


where $\theta(u) \equiv 1$ if $u>0$ and $\theta(u) \equiv 0$ if $u<0$. Clearly, the interaction term has changed the paths that contribute to the path integral. This result implies that any attempt at a perturbation series must be taken about the pseudofree theory because if it is taken about the free theory every term will diverge! Domains matter!

\section{Ultralocal scalar model: a nonrenormalizable example}

The classical Hamiltonian for this model is given by

$$
H(\pi, \phi)=\int\left\{\frac{1}{2}\left[\pi(x)^{2}+m_{0}^{2} \phi(x)^{2}\right]+g_{0} \phi(x)^{4}\right\} d^{s} x,
$$

with the Poisson bracket $\left\{\phi(x), \pi\left(x^{\prime}\right)\right\}=\delta\left(x-x^{\prime}\right)$. This model has also been discussed before, e.g., [4-6], and we will offer just an overview of its quantization.

Let us first introduce an $s$-dimensional spatial lattice for (3.1) of step $a>0$ to approximate the spatial integral, which leads to the expression for a regularized classical Hamiltonian given by

$$
H_{K}(\pi, \phi)=\sum_{k}\left[\frac{1}{2}\left(\pi_{k}^{2}+m_{0}^{2} \phi_{k}^{2}\right)+g_{0} \phi_{k}^{4}\right] a^{s},
$$

where $k=\left\{k_{1}, k_{2}, \ldots, k_{s}\right\} \in K, k_{j} \in \mathbb{Z} \equiv\{0, \pm 1, \pm 2, \ldots\}$, where $K$ is large but finite and $a^{s}$ represents a cell volume. Clearly, a suitable limit of $K \rightarrow \infty$ and $a \rightarrow 0$ is understood such that $K a^{s} \rightarrow V$, where $V \leq \infty$ is the spatial volume implicit in (3.1).

Next we discuss a quantization based on CQ for the lattice Hamiltonian prior to taking a spatial limit with $a \rightarrow 0$. Since there is no connection of the dynamics between spatial points, it follows that at each lattice site $\pi_{k} \rightarrow a^{-s} P_{k}$ and $\phi_{k} \rightarrow Q_{k}$ and $H_{k}\left(\pi_{k}, \phi_{k}\right) \rightarrow \mathcal{H}_{k}\left(P_{k}, Q_{k}\right)$. Moreover, at each site $\mathcal{H}_{k}\left(P_{k}, Q_{k}\right)=\left\{\frac{1}{2}\left[a^{-2 s} P_{k}^{2}+m_{0}^{2} Q_{k}^{2}\right]+\right.$ $\left.g_{0} Q_{k}^{4}+\mathcal{O}\left(\hbar ; P_{k}, Q_{k} ; a\right)\right\} a^{s}$, where we assume the last term is polynomial in $P$ and $Q$ and vanishes if $\hbar \rightarrow 0$. Here the ground state $\left|\psi_{0 ; k}\right\rangle$ fulfills the relation $\mathcal{H}_{k}\left(P_{k}, Q_{k}\right)\left|\psi_{0 ; k}\right\rangle=0$. The normalized Schrödinger representation $\left\langle\phi_{k} \mid \psi_{0 ; k}\right\rangle$ has the form $\exp \left[-Y\left(\phi_{k} ; \hbar, a\right) a^{s} / 2\right]$, where $-\infty<Y\left(\phi_{k} ; \hbar, a\right)=Y\left(-\phi_{k} ; \hbar, a\right)<\infty$, and the characteristic function of the regularized overall ground-state distribution is given by

$$
\begin{aligned}
C_{K}(f) & =\Pi_{k} \int e^{i f_{k} \phi_{k} a^{s} / \hbar} e^{-Y\left(\phi_{k} ; \hbar, a\right) a^{s}} d \phi_{k} / \int e^{-Y\left(\phi_{k} ; \hbar, a\right) a^{s}} d \phi_{k} \\
& =\Pi_{k} \int e^{i f_{k} \phi_{k}} e^{-Y\left(\phi_{k} \hbar / a^{s} ; \hbar, a\right) a^{s}} d \phi_{k} / \int e^{-Y\left(\phi_{k} \hbar / a^{s} ; \hbar, a\right) a^{s}} d \phi_{k} .
\end{aligned}
$$

The final issue involves taking the continuum limit as $a \rightarrow 0$. To ensure a reasonable limit it is helpful to expand the term involving $f_{k}$ in a power series, which leads to

$$
C_{K}(f)=\Pi_{k}\left\{1-\frac{1}{2 !} f_{k}^{2}\left\langle\phi_{k}^{2}\right\rangle_{a}+\frac{1}{4 !} f_{k}^{4}\left\langle\phi_{k}^{4}\right\rangle_{a}-\frac{1}{6 !} f_{k}^{6}\left\langle\phi_{k}^{6}\right\rangle_{a}-\ldots\right\} .
$$

In order to achieve a meaningful continuum limit, it is necessary that $\left\langle\phi_{k}^{2}\right\rangle_{a} \propto a^{s}$, which, in the present case, means that $\left\langle\phi_{k}^{2 j}\right\rangle_{a} \propto a^{s j}$ (since the distribution is 'tall and narrow' about 
$\left.\phi_{k}=0\right)$, leading to the result that the continuum limit becomes $C(f)=\exp \left[-R \int f(x)^{2} d^{s} x\right]$ for some $R, 0<R<\infty$. This result is a standard result of the Central Limit Theorem [7], which implies a Gaussian (= free) ground state for CQ.

Next we introduce classical affine fields $\phi(x)$ and $\kappa(x)$, and the classical Hamiltonian becomes

$$
H^{\prime}(\kappa, \phi)=\int\left\{\frac{1}{2}\left[\kappa(x) \phi(x)^{-2} \kappa(x)+m_{0}^{2} \phi(x)^{2}\right]+g_{0} \phi(x)^{4}\right\} d^{s} x .
$$

A quantum study begins by promoting the classical affine fields to operators $\kappa(x) \rightarrow \hat{\kappa}(x)$ and $\phi(x) \rightarrow \hat{\phi}(x)$, and it formally follows that

$$
\hat{\kappa}(x) \hat{\phi}(x)^{-2} \hat{\kappa}(x)=\hat{\pi}(x)^{2}+\frac{3}{4} \hbar^{2} \delta(0)^{2} \hat{\phi}(x)^{-2} .
$$

On the same spatial lattice as before, the quantum Hamiltonian is chosen as

$$
\mathcal{H}_{K}^{\prime}(\hat{\kappa}, \hat{\phi})=\sum_{k}\left\{\frac{1}{2}\left[-a^{-2 s} \hbar^{2} \partial^{2} / \partial \phi_{k}^{2}+a^{-2 s} F \hbar^{2} \phi_{k}^{-2}+m_{0}^{2} \phi_{k}^{2}\right]+g_{0} \phi_{k}^{4}\right\} a^{s},
$$

where $F \equiv\left(\frac{1}{2}-b a^{s}\right)\left(\frac{3}{2}-b a^{s}\right)$ is a regularized version of $\frac{3}{4}$, and $b$ is a fixed constant so that $b a^{s}$ is dimensionless. The ground state $\psi_{0}(\phi)$ satisfies $\mathcal{H}_{K}^{\prime}(\hat{\kappa}, \hat{\phi}) \psi_{0}(\phi)=0$ and, for $b a^{s} \ll 1$, has a form such that

$$
\psi_{0}(\phi)^{2}=\Pi_{k}\left(b a^{s}\right) e^{-Z\left(\phi_{k} ; \hbar, a\right) a^{s}}\left|\phi_{k}\right|^{-\left(1-2 b a^{s}\right)} .
$$

The exponent $Z$ prevents divergence of an integral of the ground-state distribution when $\left|\phi_{k}\right| \gg 1$, but when $\left|\phi_{k}\right| \ll 1$ an integral of the ground-state distribution is proportional to $\left(b a^{s}\right)^{-1}+\mathcal{O}(1)$, in which case the pre-factor $\left(b a^{s}\right)$, when very tiny, provides the overall normalization. Once again we study the characteristic function

$$
\begin{aligned}
C(f) & =\lim _{a \rightarrow 0} \Pi_{k} \int\left\{e^{i f_{k} \phi_{k} a^{s} / \hbar}\left(b a^{s}\right) e^{-Z\left(\phi_{k} ; \hbar, a\right) a^{s}}\left|\phi_{k}\right|^{-\left(1-2 b a^{s}\right)} d \phi_{k}\right\} \\
& =\lim _{a \rightarrow 0} \Pi_{k}\left\{1-\left(b a^{s}\right) \int\left[1-e^{i f_{k} \phi_{k} a^{s} / \hbar}\right] e^{-Z\left(\phi_{k} ; \hbar, a\right) a^{s}}\left|\phi_{k}\right|^{-\left(1-2 b a^{s}\right)} d \phi_{k}\right\} \\
& =\exp \left\{-b \int d^{s} x \int\left[1-e^{i f(x) \lambda / \hbar}\right] e^{-z(\lambda ; \hbar)} d \lambda /|\lambda|\right\}
\end{aligned}
$$

where $\lambda=\phi_{k} a^{s}$, and suitable factors in $Z$ undergo an operator product expansion renormalization which leads to $z$. Besides a Gaussian distribution found by CQ, the resultant form (3.9) is the only other result of the Central Limit Theorem, namely, a (generalized) Poisson distribution (based on the new moments $\left\langle\phi_{k}^{2 j}\right\rangle_{a} \propto a^{s}$ for all $j \in\{1,2,3, \ldots\}$ ). Moreover, the classical limit as $\hbar \rightarrow 0$ for this solution has been shown [1] to yield the starting classical Hamiltonian.

As an example, suppose we take the limit $g_{0} \rightarrow 0$. In this case, $c(\lambda)=|\lambda|^{-1 / 2} e^{-b m \lambda^{2} / 2 \hbar}$, and thus

$$
\mathfrak{h}(\partial / \partial \lambda, \lambda)=\frac{1}{2} b^{-1}\left[-\hbar^{2} \partial^{2} / \partial \lambda^{2}+\frac{3}{4} \hbar^{2} \lambda^{-2}+b^{2} m^{2} \lambda^{2}\right] .
$$


The resultant characteristic function is given by

$$
C_{0}(f)=\exp \left\{-b \int d^{s} x \int\left[1-e^{i f(x) \lambda / \hbar}\right] e^{-b m \lambda^{2} / \hbar} d \lambda /|\lambda|\right\}
$$

which does not represent a traditional free model. However, the ground state implicitly described here reflects the fact that the domain of the classical free action functional is strictly larger than the domain of the classical interacting action functional and which is unchanged in the limit of the coupling constant $g_{0} \rightarrow 0$. Just as with the 'toy' example in section 2 , and from a path integral viewpoint, this domain modification leads to a different set of paths than in the free-model path integral. Moreover, the spectrum of the relevant quantum Hamiltonian associated with (3.11) has a uniform spacing $(2 \hbar m) j$, where $j \in\{0,1,2,3, \cdots\}$, and which, as promised, correctly reflects a vanishing zero-point energy [4].

This last reference also discusses different singular terms that are $\mathcal{O}\left(\hbar^{2}\right)$, which may be relevant in alternative applications.

\section{Additional studies using affine variables}

Enhanced quantization and affine variables have also been applied to other problems. The use of affine variables to discuss idealized cosmological models $[8,9]$ has led to gravitational bounces rather than an initial singularity of the universe. Further use of affine variables applied to idealized gravitational models has been given in seveal papers, e.g., [10-13], and references therein. More complex applications include covariant scalar fields [1, 14-17], and quantum gravity $[1,18-20]$, for which confirmation requires multiple selected computer simulations.

\section{Acknowledgments}

The referee is thanked for several useful corrections and helpful suggestions.

Open Access. This article is distributed under the terms of the Creative Commons Attribution License (CC-BY 4.0), which permits any use, distribution and reproduction in any medium, provided the original author(s) and source are credited.

\section{References}

[1] J.R. Klauder, Enhaced Quantization: Particles, Fields 83 Gravity, World Scientfic, Singapore (2015).

[2] C. Itzykson and J.-B. Zuber, Quantum Field Theory, McGraw-Hill, New York (1980).

[3] B. Simon, Quadratic Forms and Klauder's Phenomenon: A Remark on Very Singular Perturbations, J. Funct. Anal. 14 (1973) 295.

[4] J.R. Klauder, Beyond Conventional Quantization, Cambridge University Press, Cambridge (2000). 
[5] J.R. Klauder, Ultralocal scalar field models, Commun. Math. Phys. 18 (1970) 307 [inSPIRE].

[6] J.R. Klauder, Ultralocal quantum field theory, Acta Phys. Austriaca Suppl. 8 (1971) 227 [INSPIRE].

[7] https://en.wikipedia.org/wiki/Central_limit_theorem.

[8] J.R. Klauder and E.W. Aslaksen, Elementary model for quantum gravity, Phys. Rev. D 2 (1970) 272 [INSPIRE].

[9] M. Fanuel and S. Zonetti, Affine Quantization and the Initial Cosmological Singularity, EPL 101 (2013) 10001 [arXiv:1203.4936] [INSPIRE].

[10] H. Bergeron, E. Czuchry, J.-P. Gazeau, P. Małkiewicz and W. Piechocki, Singularity avoidance in a quantum model of the Mixmaster universe, Phys. Rev. D 92 (2015) 124018 [arXiv: 1501.07871] [INSPIRE].

[11] H. Bergeron, E. Czuchry, J.-P. Gazeau, P. Małkiewicz and W. Piechocki, Smooth quantum dynamics of the mixmaster universe, Phys. Rev. D 92 (2015) 061302 [arXiv:1501.02174] [INSPIRE].

[12] H. Bergeron, E. Czuchry, P. Malkiewicz and J.-P. Gazeau, Nonadiabatic bounce and an inflationary phase in the quantum mixmaster universe, Phys. Rev. D 93 (2016) 124053 [arXiv: 1511.05790] [INSPIRE].

[13] H. Bergeron, E. Czuchry, J.-P. Gazeau and P. Małkiewicz, Vibronic framework for quantum mixmaster universe, Phys. Rev. D 93 (2016) 064080 [arXiv:1512.00304] [INSPIRE].

[14] J.R. Klauder, Enhanced Quantization: A Primer, J. Phys. A 45 (2012) 285304 [arXiv: 1204.2870] [INSPIRE].

[15] J.R. Klauder, Enhanced Quantum Procedures that Resolve Difficult Problems, Rev. Math. Phys. 27 (2015) 1530002 [arXiv:1206.4017] [INSPIRE].

[16] J.R. Klauder, Nontrivial quantization of $\phi_{n}^{4}, n \geq 2$, Theor. Math. Phys. 182 (2015) 83 [arXiv: 1405.0332] [INSPIRE].

[17] J.R. Klauder, Scalar Field Quantization Without Divergences In All Spacetime Dimensions, J. Phys. A 44 (2011) 273001 [arXiv:1101.1706] [InSPIRE].

[18] J.R. Klauder, Noncanonical quantization of gravity. I. foundations of affine quantum gravity, J. Math. Phys. 40 (1999) 5860 [gr-qc/9906013] [INSPIRE].

[19] J.R. Klauder, Noncanonical quantization of gravity. 2. Constraints and the physical Hilbert space, J. Math. Phys. 42 (2001) 4440 [gr-qc/0102041] [INSPIRE].

[20] J.R. Klauder, Recent Results Regarding Affine Quantum Gravity, J. Math. Phys. 53 (2012) 082501 [arXiv:1203.0691] [inSPIRE]. 